\title{
Biosafety Evaluation of Equine Umbilical Cord-Derived Mesenchymal Stromal Cells by Systematic Pathogen Screening in Peripheral Maternal Blood and Paired UC-MSCs
}

\author{
Marie Denys, Albertine Léon,, ${ }^{2,3}$ Clément Robert, ${ }^{4}$ Nathalie Saulnier, ${ }^{4}$ Anne Josson-Schramme, \\ Loïc Legrand, ${ }^{2,5}$ Laurence Wimel, ${ }^{6}$ Stéphane Maddens, ${ }^{4,{ }^{*}}$ and Stéphane Pronost ${ }^{2,5,{ }^{*}}$
}

\begin{abstract}
Background: The growing interest in mesenchymal stromal cells (MSCs) in equine medicine, together with the development of MSC biobanking for allogeneic use, raises concerns about biosafety of such products. MSCs derived from umbilical cord (UC) carry an inherent risk of contamination by environmental conditions and vertical transmission of pathogens from broodmares. There is yet no report in the scientific literature about horses being contaminated by infected MSC products, and no consensus about systematic infectious screening of umbilical cord-derived mesenchymal stromal cells (UC-MSCs) to ensure microbiological safety of therapeutic products.

Objectives: To develop a standard protocol to ensure UC-MSC microbiological safety and to assess the risk of vertical transmission of common intracellular pathogens from broodmares to paired UC-MSCs.

Study Design and Methods: Eighty-four UC and paired peripheral maternal blood (PMB) samples were collected between 2014 and 2016. Sterility was monitored by microbiological control tests. Maternal contamination was tested by systematical PMB PCR screening for 14 pathogens and a Coggins test. In case of a PCR-positive result regarding one or several pathogen(s) in PMB, a PCR analysis for the detected pathogen(s) was then conducted on the associated UC-MSCs.

Results: Ten out of 84 UC samples were contaminated upon extraction and 6/84 remained positive in primo culture. The remaining 78/84 paired PMB \& UC-MSC samples were evaluated for vertical transmission; 37/78 PMB samples were PCR positive for Equid herpesvirus (EHV)-1, EHV-2, EHV-5, Theileria equi, Babesia caballi, and/or Mycoplasma spp. Hepacivirus was detected in 2/27 cases and Theiler Diseases Associated Virus in 0/27 cases (not performed on all samples due to late addition). All paired UC-MSC samples tested for the specific pathogen(s) detected in PMB were negative (37/37).

Main Limitations: More data are needed regarding MSC susceptibility to most pathogens detected in PMB. Conclusions: In-process microbiological controls combined with PMB PCR screening provide a comprehensive assessment of UC-MSC exposure to infectious risk, vertical transmission risk appearing inherently low.
\end{abstract}

Keywords: horse, mesenchymal stem cells, cellular therapy, vertical transmission, PCR, biosecurity

\section{Introduction}

I

N EQUINE VETERINARY MEDICINE, the use of mesenchymal stromal cells (MSCs) for regenerative purposes is expanding steadily, with clinical safety now established, whether used in an autologous or allogeneic setting. ${ }^{1}$ However, the biosafety infectious profile of MSCs has been poorly reported so far, although they carry a potential infectious risk originating from the donor or the biomanufacturing process and cannot be completely sterilized. Compared with autologous use, the risk is higher with allogeneic MSCs since products manufactured from a single donor can be administered to several recipients. Reports about iatrogenic contamination with blood-derived products exist. ${ }^{2,3}$

\footnotetext{
${ }^{1}$ VetAgro Sup, Université de Lyon, Marcy l'Etoile, France.

${ }^{2}$ LABEO Frank Duncombe, Saint Contest, France.

${ }^{3}$ U2RM, Normandie Univ, UNICAEN, Caen, France.

${ }^{4}$ Vetbiobank SAS, Marcy l'Etoile, France.

${ }^{5}$ BIOTARGEN, Normandie Univ, UNICAEN, Caen, France.

${ }^{6}$ Experimental Farm, French Horse and Riding Institute (IFCE), Chamberet, France.

*These authors contributed equally to this work.
} 
The European Medicines Agency (EMA)'s Committee for Medicinal Products for Veterinary Use (CVMP) developed an expert group for novel therapy-related issues named ADVENT (Ad Hoc Expert Group on Veterinary Novel Therapies), aiming to provide guidelines strengthening quality and security. It recently released two question and answer documents relative to sterility ${ }^{4}$ and freedom from extraneous agents ${ }^{5}$ of allogeneic MSC products, with recommendations based on product specificities and epidemiological considerations. These guidance documents cover biosafety aspects that must be considered when manufacturing stem cell-based products for allogeneic use, according to the requirement to test veterinary medicinal products for potential infectious contaminants specified in Directive 2001/82/EC, Regulation (EU) 2019/6. ${ }^{5}$

Similarly, in the United States, cell-based drugs must be reviewed and approved by the Food and Drug Administration following Center for Veterinary Medicine's (CVM) guidance before they can be legally marketed. ${ }^{6}$ This article provides a precise description of a protocol defined on a risk-based analysis, integrating the existing regulatory recommendations.

It is acknowledged that the various anatomical structures from which MSCs can be isolated (UC, bone marrow, and so on) do not present the same risk: human umbilical cord-derived mesenchymal stromal cells (UC-MSCs) are considered less likely to contain infectious agents than MSCs derived from adult tissues. ${ }^{7}$ No data have been published in the equine veterinary context yet. Equids also present a high risk of environmental contamination during UC sampling. Therefore, we conducted a systematic evaluation of bacteriological and fungal changes upon initiation of biomanufacturing and before freezing of master bank MSC units. Although the equine epitheliochorial placentation reduces risk of transplacental transmission of common pathogens, vertical transmission has been demonstrated for some of them, such as piroplasmosis or classical abortive viruses like Equid herpesvirus (EHV)-1 and equine arteritis virus, ${ }^{8,9}$ or recently hepacivirus, ${ }^{10}$ whereas evidence-based information is missing for others.

To assess the risk of vertical contamination of UC-MSCs, we carried out a systematic screening on peripheral maternal blood (PMB) samples from healthy broodmares whose UC was collected for MSCs. Fifteen equine intracellular agents of interest ${ }^{11}$ were selected based on four criteria adapted to the French epidemiological situation: endemic microorganisms (Babesia caballi, Theileria equi, EHV-1, EHV-2, EHV-4, and EHV-5, Rhodococcus equi, Anaplasma phagocytophilum, Borrelia burgdorferi, and Leptospira interrogans), notifiable diseases of importance and high pathogenicity (equine infectious anemia and equine viral arteritis), emerging pathogens (hepacivirus and Theiler Diseases Associated Virus [TDAV]), and identified risks of unknown relevance (Mycoplasma spp.).

All those data will support a better assessment of UCMSC-specific infectious risk for allogeneic use and will help in defining the donor qualification strategy.

\section{Materials and Methods}

\section{Experimental cohort}

This protocol was conducted on 84 foals between breeding seasons during 2014 and 2016. Broodmares were sta- tioned at the experimental farm of French Horse and Riding Institute (IFCE, Chamberet, France) or in privately owned high-standard French stud farms and benefitted from medical follow-up by qualified equine veterinarians. Two weeks before expected parturition, broodmares were examined for clinical signs of systemic or urogenital disease. In the absence of abnormalities, mares were considered healthy and prepared to foaling according to farm protocols, with adequate monitoring.

\section{Tissue and biological fluid collection}

PMB samples were collected immediately after foaling (one tube with EDTA and one tube without anticoagulant). Trained staff were in charge of the collection of umbilical cord tissue (UCT) $(n=80)$ or umbilical cord blood (UCB) $(n=4)$, according to standardized protocols that have been proven harmless to both foal and mare. ${ }^{12}$ UCT samples were serially washed with sterile physiological solution and conditioned in storage medium complemented with $2 \%$ (v/v) antibiotic solution (penicillin 10,000 U/mLstreptomycin $10 \mathrm{mg} / \mathrm{mL}$-amphotericin B $25 \mu \mathrm{g} / \mathrm{mL}$ ). UCB samples were collected through clean venipuncture in $250 \mathrm{~mL}$ collection bags with anticoagulant, serving as containers for shipment. Within 24-48 hours after foaling, all samples were shipped to the biomanufacturing laboratory using single-use material to ensure temperature between $+2^{\circ} \mathrm{C}$ and $+12^{\circ} \mathrm{C}$. Umbilical samples were processed and PMB tubes redirected to control quality laboratory for pathogen screening according to flowchart (Fig. 1a).

\section{UC-MSC isolation and culture}

Processing of UCT. UCT were submitted to serial washings using sterile $\mathrm{NaCl} 0.9 \%$, soaked in povidone iodine for 15 minutes, and dissected with single-use material to extract the conjunctive tissue surrounding umbilical vessels (Wharton's jelly). Dissected tissue was minced and digested for $\sim 1$ hour with collagenase type I (SigmaAldrich) at $37^{\circ} \mathrm{C}$, to allow cell extraction.

The suspension of digested tissue was sampled for environmental contamination testing (see During MSC Extraction section). Suspension of digested tissue was plated into Falcon ${ }^{\circledR}$ cell culture flasks with growth medium, consisting of DMEM supplemented with $100 \mathrm{U} / \mathrm{mL}$ Penicillin/ Streptomycin, $2 \mathrm{mM}$ L-glutamine, and 10\% (v/v) HyClone ${ }^{\circledR}$ fetal calf serum.

Processing of $U C B$. As previously described, ${ }^{13}$ whole UCB samples were loaded onto a Ficoll $(1.077 \mathrm{~g} / \mathrm{mL}$ density) solution. After density gradient centrifugation at $900 \mathrm{~g}$ for 30 minutes at room temperature, the cellular suspension was sampled for environmental contamination testing (see During MSC Extraction section). Mononuclear cells were removed from the interphase and washed twice with Dulbecco's phosphate-buffered saline (D-PBS), plated at a defined seeding density $\left(150,000\right.$ cells $\left./ \mathrm{cm}^{2}\right)$ into Falcon cell culture flasks, and cultured as for UCT-MSCs.

Culture and storage of isolated UCT-MSCs \& UCB-MSCs before screening. After 72 hours, nonadherent cells were eliminated by changing the medium. After enzymatic detachment with trypsin $0.05 \%$ /EDTA $0.02 \%$ in PBS, adherent cells were reseeded and allowed to expand until $80 \%$ confluence, harvested, washed to eliminate antibiotics, and 
sampled for microbiology testing (see After MSC Expansion section). Finally, UC-MSCs were conditioned for freezing at $1.5 \times 10^{6}$ cells/unit in DMEM supplemented with $10 \%(\mathrm{v} / \mathrm{v})$ FBS and $10 \%$ (v/v) DMSO and stored at $-196^{\circ} \mathrm{C}$. Specific units were frozen for future PCR screening.

All cell culture reagents were purchased from Pan Biotech (PAN-Biotech, Aidenbach, Germany) unless otherwise specified.

\section{Environmental contamination testing}

Environmental contamination was monitored throughout the manufacturing process to detect microorganisms coming from raw material (UC sampling conditions) or biomanufacturing process (laboratory contamination) as described in Figure 1a.

During MSC extraction. Samples from digested tissue (UCT) or cellular solution (UCB) were tested with a broadspectrum test (brain heart infusion $[\mathrm{BHI}]$; bioMérieux, Marcy l'Etoile, France), incubated for 7 days at $37^{\circ} \mathrm{C}$, and checked daily for visual turbidity.

After MSC expansion. Aliquots from prefreezing cell suspension were also tested with a broad-spectrum test $(\mathrm{BHI}$; bioMérieux), incubated for 7 days at $37^{\circ} \mathrm{C}$, and checked daily for visual turbidity. Moreover, on day 5, a sample from BHI suspension was plated on Columbia Agar $+5 \%$ sheep

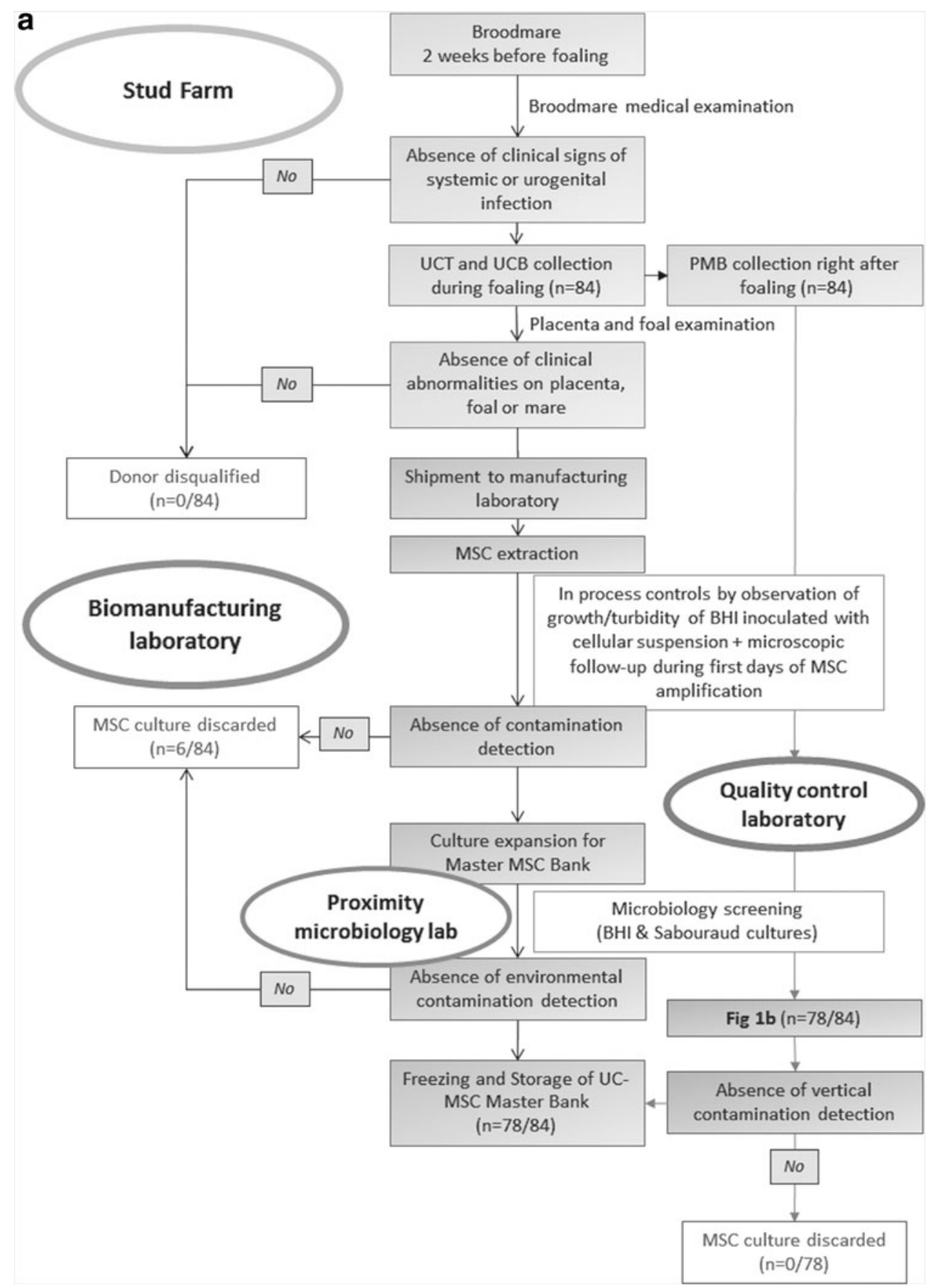

FIG. 1. Flowchart describing (a) Steps and controls from donor qualification to freezing of MSC Master Bank; (b) paired PMB and UC-MSC (dotted line) screening by quality control laboratory. BHI, brain heart infusion; MSC, mesenchymal stromal cell; PMB, peripheral maternal blood; UCB, umbilical cord blood; UC-MSCs, umbilicalcord-derived mesenchymal stromal cells; UCT, umbilical cord tissue. 


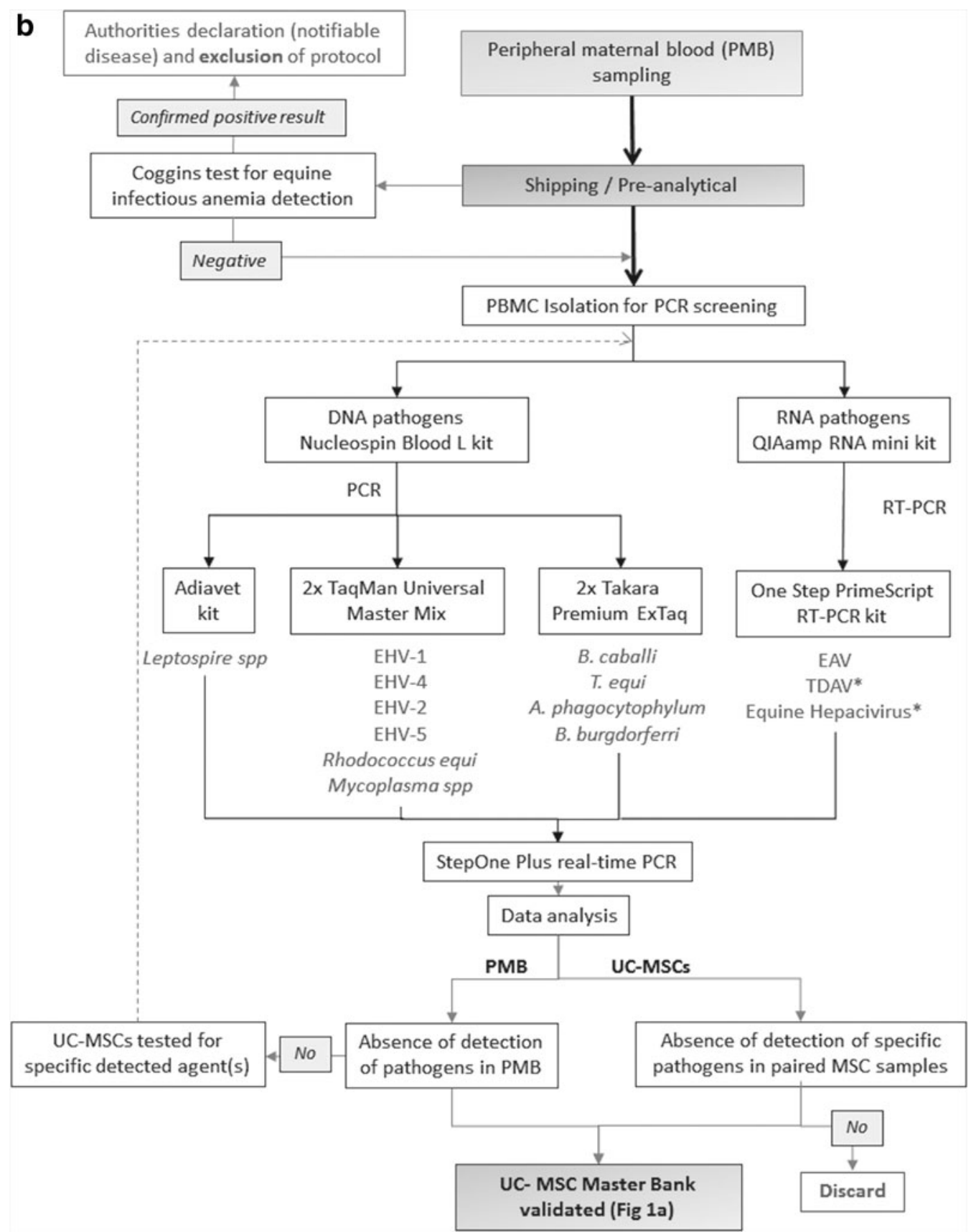

FIG. 1. (Continued).

blood (bioMérieux) and examined for bacterial colony growth proximity (LVD69, Marcy l'Etoile, France). Since 2015, an additional aliquot had been seeded on Sabouraud Dextrose Chloramphenicol Agar (bioMérieux) incubated at $+20^{\circ} \mathrm{C}$ for 10 days.

Results were considered positive when microorganism development was visually detected. Further identification of colonies on these plates was not pursued.

\section{PMB screening and analysis on paired UC-MSCs}

All 78 samples that were free of detectable fungal or bacterial contamination were submitted to a Coggins test and PCR screening on PMB. Hepacivirus and TDAV
PCR were developed after the beginning of the study and performed on only 27 PMB samples. When positive results were obtained for one or several agents, paired UCMSCs were retrieved from nitrogen storage, thawed, washed from cryoprotectant, conditioned at $1-2.5 \times 10^{6}$ cells/mL in a DNA/RNA stabilizing RNAse-free solution, and tested for the specific detected pathogens (Fig. 1b).

PCR tests. Concerning DNA pathogens, nucleic acids were extracted from $2 \mathrm{~mL}$ of each sample with the $\mathrm{Nu}$ cleospin Blood L kit (Macherey-Nagel, Düren, Germany), then eluted in a final volume of $200 \mu \mathrm{L}$, and stored at $-80^{\circ} \mathrm{C}$ until used. Concerning RNA viruses, nucleic acids were extracted from $140 \mu \mathrm{L}$ of each sample with the QIAamp ${ }^{\circledR}$ 
Table 1. Sequences of Primers and Probes Used in This Study

\begin{tabular}{|c|c|c|c|c|}
\hline Pathogens & $\begin{array}{l}\text { Name of primer } \\
\text { or probe }\end{array}$ & Nucleotide sequence (5'-3') & $\begin{array}{l}\text { Size } \\
(b p)\end{array}$ & Reference \\
\hline \multirow[t]{3}{*}{ EAV } & EAV7.53F & GGCGACAGCCTACAAGCTACA & \multirow[t]{3}{*}{204} & \multirow[t]{3}{*}{18} \\
\hline & EAV7.53R & CGGCATCTGCAGTGAGTGA & & \\
\hline & EAV7.53 probe & FAM-TTGCGGACCCGCATCTGACCAA-TAMRA & & \\
\hline \multirow[t]{3}{*}{ EHV1 } & EHV-1 MGB F1 & CATGTCAACGCACTCCCA & \multirow{3}{*}{63} & \multirow[t]{3}{*}{33} \\
\hline & EHV-1 MGB R1 & GGGTCGGGCGTTTCTGT & & \\
\hline & EHV-1MGB & FAM-CCCTACGCTGCTCC-MGB-NFQ & & \\
\hline \multirow[t]{3}{*}{ EHV2 } & EHV-2 gB F & GTGGCCAGCGGGGTGTTC & \multirow[t]{3}{*}{100} & \multirow[t]{3}{*}{15} \\
\hline & EHV-2 gB R & CCCCCAAAGGGATTYTTGAA & & \\
\hline & $\begin{array}{l}\text { EHV-2 } \mathrm{gB} \\
\text { probe }\end{array}$ & FAM-CCCTCTTTGGGAGCATAGTCTCGGGG-MGB-NFQ & & \\
\hline \multirow[t]{3}{*}{ EHV4 } & EHV-4 MGB F1 & GGGCTATTGGATTACAGCGAGAT & \multirow[t]{3}{*}{58} & \multirow[t]{3}{*}{34} \\
\hline & EHV-4 MGB R1 & TAGAATCGGAGGGCGTGAAG & & \\
\hline & $\begin{array}{l}\text { EHV-4 MGB } \\
\text { Probe }\end{array}$ & VIC-CAGCGCCGTAACCAG-MGB-NFQ & & \\
\hline \multirow[t]{3}{*}{ EHV5 } & EHV-5 gB F & AACCCGCCGTGCATCA & \multirow[t]{3}{*}{66} & \multirow[t]{3}{*}{15} \\
\hline & EHV-5 gB R & AGGCGCCACACACCCTAA & & \\
\hline & $\begin{array}{l}\mathrm{EHV}-5 \mathrm{gB} \\
\text { probe }\end{array}$ & FAM-ACAACACCACCAACCCCTTTCTGCTG MGB-NFQ & & \\
\hline \multirow[t]{5}{*}{ Hepacivirus } & Qanti-5UF1 & GAGGGAGCTGGAATTCGTGAA & \multirow[t]{5}{*}{157} & \multirow[t]{4}{*}{35} \\
\hline & Qanti-5UR1 & GCAAGCATCCTATCAGACCGT & & \\
\hline & Sau5UF & TCGAGGGAGCTGRAATTCGT & & \\
\hline & Sau5UR & GCCCTCGCAAGCATCCTATC & & \\
\hline & Qanti-5UProbe & FAM-CCACGAAGGAAGGCGGGGGC-BHQ1 & & \multirow{3}{*}{36} \\
\hline \multirow{3}{*}{ TDAV } & EVT-146b & AGGGTTCTTCGGGTAAATCCC & \multirow[t]{3}{*}{165} & \\
\hline & EVT-147b & CCTCGGACTGAATTATAGGCGT & & \\
\hline & TCHA P 5 & FAM-CGCTGGTTCCTGGTACCACCACCC-BHQ1 & & \multirow{3}{*}{37} \\
\hline \multirow{3}{*}{$\begin{array}{l}\text { Rhodococcus } \\
\quad \text { equi }\end{array}$} & REqui-F & CAGCAGTGCGATTCTCAATAGTG & \multirow[t]{3}{*}{75} & \\
\hline & REqui-R & GAAGTCGTCGAGCTGTCATAGCT & & \\
\hline & REqui-P & FAM-CAGAACCGACAATGCCACTGCCTG-BHQ1 & & \\
\hline $\begin{array}{l}\text { Leptospira } \\
\quad \text { interrogans }\end{array}$ & & ADIAVET $^{B}$ LEPTO REAL TIME (BioX) $^{2}$ & NA & 38 \\
\hline Mycoplasma spp. & GPO-3 & GGGAGCAAACAGGATTAGATACCCT & 160 & 39 \\
\hline & MGSO-D & TGCACCAYCTGTCAYTCYGTTAACCTC & & \\
\hline & Yak & YakimaYellow-CCGCACAAGCGGTGGAGCATG-BHQ1 & & \\
\hline Theileria equi & Be18SF & GCGGTGTTTCGGTGATTCATA & 81 & 40,41 \\
\hline & Be18SR & TGATAGGTCAGAAACTTGAATGATACATC & & \\
\hline & Be18SS & VIC-AAATTAGCGAATCGCATGGC-MGB-NFQ & & \\
\hline Anaplasma & ANAPHA-F & TTTGATTCGGGGTCGAAAAA & 122 & Unpublished \\
\hline phagocytophilum & ANAPHA-R & AACGCTTCAACAGCCTCACG & & data \\
\hline & ANAPHA-P & FAM-TCGCCCTAAAGCACCAGAGGATCTG-MGB-NFQ & & \\
\hline Borrelia & SPint-F & CGCAGCTTACCACGACCTTC & 282 & 42 \\
\hline burgdorferi & SPint-R & GAGTTCGCGGGAGAGTAGGTT & & \\
\hline & SPint-P & FAM-TGGTGAATGCCTAGGAGCTTTAAGGCG-BHQ1 & & \\
\hline Babesia caballi & $\mathrm{Bc} 18 \mathrm{SF}$ & GTAATTGGAATGATGGCGACTTAA & 95 & 40 \\
\hline & Bc18SR & CGCTATTGGAGCTGGAATTACC & & \\
\hline & $\mathrm{Bc} 18 \mathrm{SP}$ & FAM-CCTCGCCAGAGTAA-MGB-NFQ & & \\
\hline
\end{tabular}

F, forward; R, reverse; $S$, sense.

EAV, equine arteritis virus; EHV, equid herpes virus; NA: not available (Diagnostic kit); TDAV, Theiler's disease associated virus.

RNA viral Mini Kit (Qiagen, Courtaboeuf, France), then eluted in a final volume of $50 \mu \mathrm{L}$, and stored at $-80^{\circ} \mathrm{C}$ until used. Five microtiter of extract was used for PCR tests, except for for EHVs, R. equi, B. caballi, and T. equi where $2.5 \mu \mathrm{L}$ of extract was used.

All primers and probes used are presented Table 1. Realtime PCRs for EHV-1, EHV-4, EHV-2, EHV-5, TDAV, equine hepacivirus, B. caballi, T. equi, R. equi, Mycoplasma spp., A. phagocytophilum, and B. burgdorferi were developed as previously described ${ }^{14,15}$ based on the standard model AFNOR NF U47-600-2. ${ }^{16,17}$ Each reaction was processed in a total volume of $25 \mu \mathrm{L}$ containing Taqman $^{\circledR}$
Universal PCR Master Mix (Life Technologies, Saint Aubin, France) for EHV-1, EHV-4, EHV-2, EHV-5, R. equi, and Mycoplasma spp. or Takara Premix Ex Taq for B. caballi, T. equi, A. phagocytophilum, and B. burgdorferi, and in a total volume of $25 \mu \mathrm{L}$ of One Step Prime Script RTPCR kit (Perfect Real time) for the TDAV and equine hepacivirus. L. interrogans kit analysis was used as described by the manufacturer (Adiagene, Ploufragan, France) and EAV PCR test performed as described previously. ${ }^{18}$ Quantitative PCR assays were performed on StepOnePlus ${ }^{\mathrm{TM}}$ RealTime PCR systems (Life Technologies). Controls in each series included extraction positive and negative controls; 
PCR positive and negative. An internal standard (HPRT1 gene, TaqMan gene expression assay; Life Technologies) was amplified to attest good extraction and absence of inhibitors. Data were analyzed manually using the StepOne ${ }^{\mathrm{TM}}$ software, version 2.2.2 (Adiagene, Ploufragan, France). All the steps were carried out by referring to the quality criteria of the AFNOR U47600 standard.

Coggins test. A Coggins test was performed on all 78 PMB serum samples according to AFNOR NF-U47-002 ${ }^{19}$ by the agar immunodiffusion technique with protein p26 incubated at $21^{\circ} \mathrm{C}+4$ for 20 hours.

\section{Results}

\section{Broad-spectrum environmental screening}

Ten of 84 UC samples were positive (turbid BHI broth) during the MSC extraction phase. After cellular culture expansion with antimicrobial complementation, 4 samples turned out negative, but 6/84 remained positive (BHI \& Sabouraud cultures), and were therefore excluded.

\section{Detection of microbial genome by PCR in PMB}

PCR screening was performed on 78/84 PMB samples, and $37 / 78$ were positive for a least one agent. Among the systematically tested pathogens, EHV-1, EHV-2, EHV-5, T. equi, and B. caballi, Mycoplasma spp. were detected at least once throughout the study, with respective prevalence of $2 / 78,22 / 78,26 / 78,7 / 78,1 / 78$, and 1/78. Co-infections were frequent (Fig. 2). Hepacivirus tested positive in $2 / 27$ cases and TDAV in 0/27 cases (not included in Fig. 2 due to late addition to protocol).

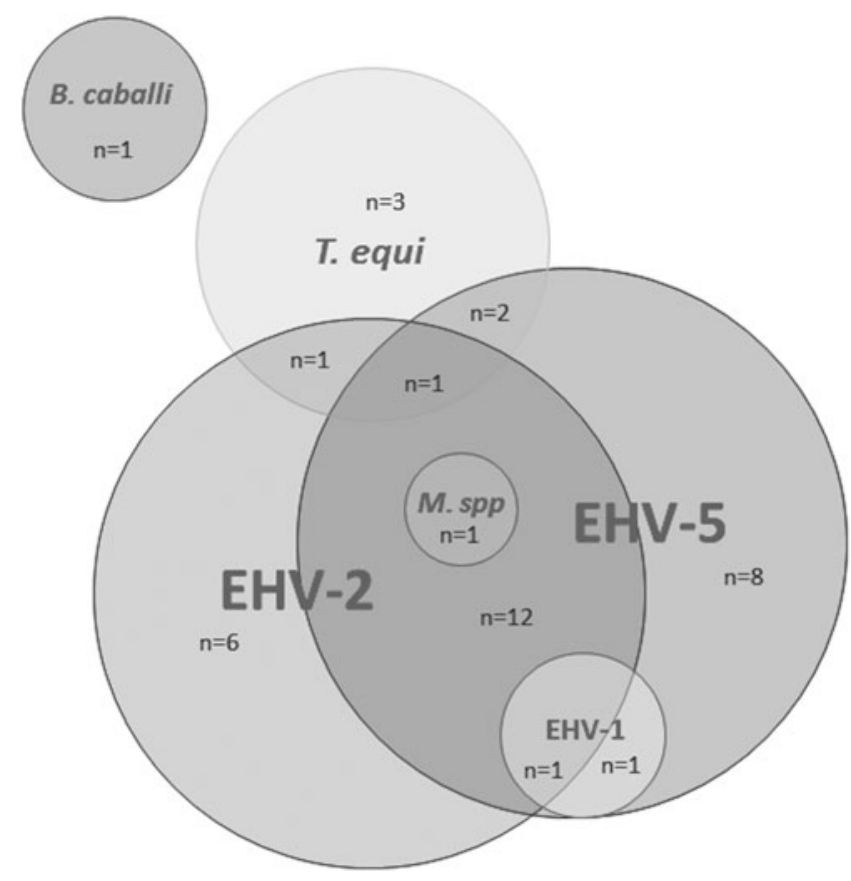

FIG. 2. Pathogens detected in peripheral maternal blood by PCR: $n$ indicates the number of positive samples. Coinfections appear with the overlap of bubbles. B. caballi, Babesia caballi; EHV-1, equid herpesvirus 1; EHV-2, equid herpesvirus 2; EHV-5, equid herpesvirus 5; M. spp, Мycoplasma spp.; T. equi, Theileria equi.

\section{Absence of detection of equine infectious anemia by Coggins test in PMB}

All PMB samples tested (78/78) were negative for equine infectious anemia.

\section{Absence of detection of intracellular pathogens in UCT and UCB-MSCs}

For every PMB sample displaying a positive PCR result regarding one or several extraneous agent(s) (37/78), paired UC-MSC units were tested negative for the specific relevant pathogen(s) (37/37). No hepacivirus was detected in the two UC-MSC samples associated with PCR-positive PMB.

\section{Discussion}

UC (tissue and blood) can suffer environmental contamination from the female genital tract and foaling conditions. To assess this risk, broad-spectrum bacteriological and fungal cultures were performed. Inherent to sampling procedures, UCT appear more prone to external contamination than $\mathrm{UCB},{ }^{12}$ although the great number difference between UCT $(n=80)$ and UCB $(n=4)$ samples in our study does not allow statistical confirmation of this. However, we highlight the importance of an early broad-spectrum test to detect contaminated material.

Donor qualification is a key element of allogenic products. The use of specific pathogen-free (SPF) herds whose infectious status is known and controlled is usually recommended. For example, the American Association of Equine Practitioners (AAEP), regarding licensed equine plasma, recommends testing donor animals against equine infectious anemia, piroplasmosis, dourine, glanders, and brucellosis. However, considering current epidemiological French status free of the last three diseases, as monitored by the RESPE (French Epidemiological Network of Equine Diseases), we decided not to include them in our panel. SPF herds are difficult and costly to operate in practice for allogenic UC-MSCs and could also raise ethical concern, hence the choice of UC collection upon usual foaling. Like in human public cord blood banking, a comprehensive qualification strategy addressing both broodmare infectious status and collected products is needed. Broodmares should be free of active infection and vaccinated, even though vaccination does not offer a full protection against infection as illustrated by EHV-1 vaccination failing to completely suppress cell viremia and its consequences. ${ }^{20}$ Samples must therefore be screened for an extensive panel of infectious agents on a risk-based analysis defined by their pathogenicity and prevalence.

Although most of the studies involving equine MSCs include a screening panel, only a few of them document the detection techniques, the list of pathogens, and the corresponding results. Previous studies reported the presence of EHV in half bone marrow samples ${ }^{21}$ and $71 \%$ of UCB samples without maternal infectious status. ${ }^{22}$ Our study provides screening results of a larger cohort, together with all the details about the detection methods. In our methodology, we tested PMB and paired MSC products as recommended by EMA, ${ }^{5}$ rather than an aliquot of UCT/UCB itself because an uneven pathogen distribution may decrease detection sensitivity. PCR techniques (excluding Coggins 
for equine infectious anemia) seem to be the most suitable methods at this stage. Indeed, the serological methods often used for screening are not recommended because the presence or absence of the genome of the pathogen is the only information of interest for the qualification of the samples. If other molecular biology methods allowing the direct detection of pathogens, such as DNA ship approaches or whole-genome sequencing, will present an alternative tomorrow, their use remains limited today, especially because it is difficult to know what to do when unknown agents are detected. Regular updating of a well-defined list for PCR screening remains an appropriate tool. The defined panel of pathogens is adapted to diseases observed in France, considering current knowledge and epizootic data, and its evolutionary aspect is illustrated by the recent addition of hepacivirus and TDAV as a guarantee of performance and security. It aims to be scalable, including other emerging pathogens in the future, like equine pegivirus 1 or maybe Neospora caninum and Coxiella burnetii recently found in equine aborted fetuses, ${ }^{23}$ suggesting vertical transmission. ${ }^{24}$

Our study showed the presence of various intracellular pathogens in PMB of clinically healthy broodmares, undetected in each UC-MSC paired sample. Two PMB samples were detected with EHV-1 together with gamma-herpesviruses (GHVs) EHV5 $(n=1)$ and EHV2-EHV5 $(n=1)$. This is consistent with the conventional thought that subclinical EHV-1 infections are common. ${ }^{9}$ The absence of clinical signs has been confirmed in our study, both in broodmares and newborn foals, and in other horses from the same barns. Subclinical infection of apparently healthy broodmares must therefore be considered, and risks of exogenous or endogenous (stress-induced viral reactivation) introduction of EHV-1 should be closely monitored in the context UC-MSC banking, especially as susceptibility of equine blood-derived MSCs for EHV-1 has been demonstrated in vitro, ${ }^{25}$ even if no EHV-1 sequence could be detected in paired UC-MSC samples in our study. In a similar approach, despite EHV-4 low prevalence (confirmed by its absence of detection), we recommend testing this agent whose variants present genetic similarities with EHV-1, since it is regularly found in respiratory diseases and is sporadically responsible for abortion (1-2 cases reported in France per year, RESPE data).

PMB screening revealed high levels of equine gammaherpesviruses EHV-2 (22/78) and EHV-5 (26/78) with 15/78 co-infected mares, consistent with a high percentage of EHV-2 and EHV-5 prevalence in France. ${ }^{15}$ GHV genome was previously found in 4/8 bone marrow samples and 5/7 cord blood samples. ${ }^{22,21}$ No GHV could be detected by PCR on any UC-MSC paired sample, arguing for a possible "privileged" status of umbilical tissue cord regarding GHV distribution. ${ }^{26}$ Our results are consistent with another study showing that some mares carrying GHV in PMB can deliver a negative fetus/foal or placenta. ${ }^{27} \mathrm{EVH}-5$ is a ubiquitous virus found in healthy and diseased horses. Even if EHV-2 pathogenicity is considered weaker, this virus also remains an infectious agent for the equine species. ${ }^{28}$ Considering this and the high prevalence of GHV in our population, we recommend future systematic PCR screening of UC-MSCs for EHV-2 and EHV-5 without a need of PMB screening for those pathogens, even if UC-MSCs appear free of equine GHV contamination for the moment.

No genome of B. caballi and T. equi was detected in UCMSC samples paired to positive PMB despite transplacental transmission to the fetus as previously described. ${ }^{29}$ Sus- ceptibility of MSCs to those two intraerythrocytic parasites is currently unknown (although a multiplication phase inside leukocytes has been described for $T$. equi). Since bone marrow is suspected to be a reservoir site of these protozoans, UC might be a safer source of MSC than bone marrow regarding these pathogens. ${ }^{30}$

Mycoplasma spp. genome was detected in one PMB sample and not in the paired UC-MSCs. Hepacivirus and TDAV detection in equine commercial serum-based products $^{3}$ motivated development of detection techniques during this study, although their pathogenicity is not well understood. PCR screening was performed on the last 27 samples. The prevalence of equine hepacivirus in PMB samples in our study (2/27) correlates with results reported by another study on 1037 French horses $(6.2 \%))^{31}$ More data are needed to assess tropism of this emerging threat toward UC-MSCs.

Endemic pathogens were unsurprisingly detected in PMB, but interestingly undetected in paired UC-MSCs. Because of the high prevalence of asymptomatic carriers among the equine population in France, including broodmares, it seems reasonable not to exclude these donors based on a broad-spectrum PCR screening. However, to fully investigate vertical transmission of these pathogens, an experimental protocol should include analyses on PMB, raw UCB or UCT, and processed UC-MSCs. Also, UCMSC samples were only tested for specific agents detected in paired PMB samples, postulating that an agent undetected in PMB would not be present in UC-MSCs. However, we may underestimate the frequency of other routes of infection of UC-MSCs (like presence of GHV in the female genital tract ${ }^{32}$ ).

In conclusion, this article provides a first comprehensive assessment of the biosafety of equine UC-MSCs for allogeneic use and cell banking. Biobanking is a dynamic process in veterinary therapy and requires a new regulatory status to ensure equine patients against iatrogenic contamination. A decisional algorithm as described in our study could be applied, considering the specific risk associated with each agent and based on screening results from both broodmare and paired UC-MSCs.

\section{Authorship}

S.M. and S.P. conceived and designed the study. Data collection was performed by M.D. and C.R. C.R. and N.S. performed MSC isolation and culture. PCR analysis and development were performed by A.L., L.L. and S.P. A.J.-S. and L.W. supervised sampling of the first umbilical cords provided and reviewed the process. The article was drafted by M.D., C.R., S.M., and S.P., and reviewed by all authors before submission.

\section{Ethical Animal Research}

Research Ethics Committee was not requested for this study.

\section{Owner Informed Consent}

All mare owners received detailed procedural information. Specific prior written consent from owners, including a retraction delay period, was obtained. 


\section{Acknowledgments}

We wish to thank Dr. Romain Paillot (LABÉO Frank Duncombe) for his kind contribution to this article, Dr. Samuel Buff and the Biological Resources Center (CRB CryAnim, member of Infrastructure CRB-Anim, ANR11INBS-0003) for providing the logistical support for the storage of biological samples, Cédric Dubois (IFCE Chamberet) for collecting umbilical cord samples, Claire Milard, Karine Groud, and Brigitte Chataigner (LVD 69) for performing broad-spectrum environmental contamination testing and reviewing this part of the article.

\section{Author Disclosure Statement}

S.M. is a current and principal shareholder of Vetbiobank. C.R. and N.S. are current employees of Vetbiobank. The remaining authors declare that the research was conducted in the absence of any commercial or financial relationships that could be construed as a potential conflict of interest.

\section{Funding Information}

Vetbiobank partially funded the study by supplying all cellular products and covering for microbiological and PCR screening costs.

\section{References}

1. Ardanaz N, Vázquez FJ, Romero A, et al. Inflammatory response to the administration of mesenchymal stem cells in an equine experimental model: Effect of autologous, and single and repeat doses of pooled allogeneic cells in healthy joints. BMC Vet Res 2016;12:65.

2. Roberts H. Equine infectious anaemia in Europe: An ongoing threat to the UK. Vet Rec 2017;181:442-446.

3. Postel A, Cavalleri J-MV, Pfaender S, et al. Frequent presence of hepaci and pegiviruses in commercial equine serum pools. Vet Microbiol 2016;182:8-14.

4. European Medicines Agency. EMA/CVMP/ADVENT/ 751229/2016. www.ema.europa.eu/en/documents/scientificguideline/questions-answers-allogenic-stem-cell-basedproducts-veterinary-use-specific-questions-sterility_en.pdf. 2017. (Last accessed March 21, 2019).

5. European Medicines Agency. EMA/CVMP/ADVENT/ 803494/2016. www.ema.europa.eu/en/documents/scientificguideline/questions-answers-allogenic-stem-cell-basedproducts-veterinary-use-specific-questions-extraneous_en .pdf. 2017. (Last accessed March 21, 2019).

6. Center for Veterinary Medicine. CVM GFI \#218 CellBased Products for Animal Use. www.fda.gov/regulatoryinformation/search-fda-guidance-documents/cvm-gfi-218-cellbased-products-animal-use. 2015. (Last accessed September 10, 2019).

7. Behzad-Behbahani A, Pouransari R, Tabei SZ, et al. Risk of viral transmission via bone marrow progenitor cells versus umbilical cord blood hematopoietic stem cells in bone marrow transplantation. Transplant Proc 2005;37:3211-3212.

8. Allsopp MTEP, Lewis BD, Penzhorn BL. Molecular evidence for transplacental transmission of Theileria equi from carrier mares to their apparently healthy foals. Vet Parasitol 2007;148:130-136.

9. Lunn DP, Davis-Poynter N, Flaminio MJBF, et al. Equine herpesvirus-1 consensus statement. J Vet Intern Med 2009; $23: 450-461$.
10. Gather T, Walter S, Todt D, et al. Vertical transmission of hepatitis $\mathrm{C}$ virus-like non-primate hepacivirus in horses. J Gen Virol 2016;97:2540-2551.

11. Sellon DC (ed). Equine Infectious Diseases. 2nd ed. St. Louis, MO: Elsevier; 2014.

12. Bartholomew S, Owens SD, Ferraro GL, et al. Collection of equine cord blood and placental tissues in 40 thoroughbred mares. Equine Vet J 2009;41:724-728.

13. Koch TG, Heerkens T, Thomsen PD, et al. Isolation of mesenchymal stem cells from equine umbilical cord blood. BMC Biotechnol 2007;7:26.

14. Doubli-Bounoua N, Richard EA, Léon A, et al. Multiple molecular detection of respiratory viruses and associated signs of airway inflammation in racehorses. Virol J 2016; 13:197.

15. Hue ES, Fortier GD, Fortier CI, et al. Detection and quantitation of equid gammaherpesviruses (EHV-2, EHV5) in nasal swabs using an accredited standardised quantitative PCR method. J Virol Methods 2014;198:18-25.

16. Hue ES, Fortier CI, Laurent AM, et al. Development and validation of a quantitative PCR method for equid herpesvirus-2 diagnostics in respiratory fluids. J Vis Exp 2016;109:e53672.

17. AFNOR. NF U47-600-2-Animal health analysis methodsPCR-Part 2: Requirements and recommandations for the development and the validation of veterinary PCR. 2015. https://www.boutique.afnor.org/norme/nf-u47-600-2/methodesd-analyse-en-sante-animale-pcr-reaction-de-polymerisation-enchaine-partie-2-exigences-et-recommandations-pour-le-devel/ article/814080/fa182883 (accessed December 23, 2019).

18. Balasuriya UBR, Leutenegger CM, Topol JB, et al. Detection of equine arteritis virus by real-time TaqMan reverse transcription-PCR assay. J Virol Methods 2002;101: 21-28.

19. AFNOR. NF U47-002-Animal health analysis methodsDetection of antibodies against Equine Infectious Anaemia by the Agar Gel Immunodiffusion Test. 2010. https:// www.boutique.afnor.org/norme/nf-u47-002/animal-healthanalysis-methods-detection-of-antibodies-against-equineinfectious-anaemia-by-the-agar-gel-immunodiffusion-test/ article/788946/fa159229 (accessed December 23, 2019).

20. Goehring LS, Wagner B, Bigbie R, et al. Control of EHV-1 viremia and nasal shedding by commercial vaccines. Vaccine 2010;28:5203-5211.

21. Branly T, Bertoni L, Contentin R, et al. Characterization and use of equine bone marrow mesenchymal stem cells in Equine Cartilage Engineering. Study of their hyaline cartilage forming potential when cultured under hypoxia within a biomaterial in the presence of BMP-2 and TGF-B1. Stem Cell Rev Rep 2017;13:611-630.

22. Desancé M, Contentin R, Bertoni L, et al. Chondrogenic differentiation of defined equine mesenchymal stem cells derived from umbilical cord blood for use in cartilage repair therapy. Int J Mol Sci 2018;19:537.

23. Leon A, Richard E, Fortier C, et al. Molecular detection of Coxiella burnetii and Neospora caninum in equine aborted foetuses and neonates. Prev Vet Med 2012;104: 179-183.

24. Antonello AM, Pivoto FL, Camillo G, et al. The importance of vertical transmission of Neospora sp. in naturally infected horses. Vet Parasitol 2012;187:367-370.

25. Claessen C, Favoreel H, Ma G, et al. Equid herpesvirus 1 (EHV1) infection of equine mesenchymal stem cells induces a pUL56-dependent downregulation of select cell surface markers. Vet Microbiol 2015;176:32-39. 
26. Yang K, Wang J, Wu M, et al. Mesenchymal stem cells detect and defend against gammaherpesvirus infection via the cGAS-STING pathway. Sci Rep 2015;5:7820.

27. Marenzoni ML, Bietta A, Lepri E, et al. Role of equine herpesviruses as co-infecting agents in cases of abortion, placental disease and neonatal foal mortality. Vet Res Commun 2013;37:311-317.

28. Kydd JH, Lunn DP, Osterrieder K. Report of the Fourth International Havemeyer Workshop on Equid Herpesviruses (EHV) EHV-1, EHV-2 and EHV-5. Equine Vet J 2019;51:565-568.

29. Sant C, Pargass I, Basu A, et al. Investigating transplacental transmission of equine piroplasmosis in thoroughbred foals in Trinidad. J Equine Vet Sci 2016;39:S98-S99.

30. Pitel P-H, Pronost S, Scrive T, et al. Molecular detection of Theileria equi and Babesia caballi in the bone marrow of asymptomatic horses. Vet Parasitol 2010;170:182-184.

31. Pronost S, Hue E, Fortier C, et al. Identification of equine hepacivirus infections in France: Facts and Physiopathological insights. J Equine Vet Sci 2016;39:S22.

32. Marenzoni ML, Sforna M, Stefanetti V, et al. Detection of equid herpesvirus type 2 and 5 DNA in uterine flushings of mares with reproductive disorders. Vet Microbiol 2014; 174:570-576.

33. Diallo IS, Hewitson G, Wright L, et al. Detection of equine herpesvirus type 1 using a real-time polymerase chain reaction. J Virol Methods 2006;131:92-98.

34. Diallo IS, Hewitson G, Wright LL, et al. Multiplex realtime PCR for the detection and differentiation of equid herpesvirus 1 (EHV-1) and equid herpesvirus 4 (EHV-4). Vet Microbiol 2007;123:93-103.

35. Pronost S, Hue E, Fortier C, et al. Prevalence of equine hepacivirus infections in France and evidence for two viral subtypes circulating worldwide. Transbound Emerg Dis 2017;64:1884-1897.

36. Chandriani S, Skewes-Cox P, Zhong W, et al. Identification of a previously undescribed divergent virus from the Fla- viviridae family in an outbreak of equine serum hepatitis. Proc Natl Acad Sci U S A 2013;110:E1407-E1415.

37. Rodríguez-Lázaro D, Lewis DA, Ocampo-Sosa AA, et al. Internally controlled real-time PCR method for quantitative species-specific detection and vapA genotyping of Rhodococcus equi. Appl Environ Microbiol 2006;72:4256-4263.

38. Rahelinirina S, Léon A, Harstskeerl RA, et al. First isolation and direct evidence for the existence of large smallmammal reservoirs of Leptospira sp. in Madagascar. PLoS One 2010;5:e14111.

39. van Kuppeveld FJ, van der Logt JT, Angulo AF, et al. Genus- and species-specific identification of mycoplasmas by $16 \mathrm{~S}$ rRNA amplification. Appl Environ Microbiol 1993; 59:655.

40. Bhoora R, Quan M, Franssen L, et al. Development and evaluation of real-time PCR assays for the quantitative detection of Babesia caballi and Theileria equi infections in horses from South Africa. Vet Parasitol 2010;168:201-211.

41. Kim C, Blanco LBC, Alhassan A, et al. Diagnostic real-time PCR assay for the quantitative detection of Theileria equi from equine blood samples. Vet Parasitol 2008;151:158-163.

42. Portnoï D, Sertour N, Ferquel E, et al. A single-run, realtime PCR for detection and identification of Borrelia burgdorferi sensu lato species, based on the hbb gene sequence. FEMS Microbiol Lett 2006;259:35-40.

Address correspondence to: Marie Denys, DVM VetAgro Sup Université de Lyon 1 avenue Bourgelat Marcy l'Etoile 69280

France

E-mail: marie.denys@vetagro-sup.fr 